\title{
Strong convergence theorems for a sequence of nonexpansive mappings with gauge functions
}

\author{
Prasit Cholamjiak, Yeol Je Cho, Suthep Suantai
}

\begin{abstract}
In this paper, we first prove a path convergence theorem for a nonexpansive mapping in a reflexive and strictly convex Banach space which has a uniformly Gâteaux differentiable norm and admits the duality mapping $j_{\varphi}$, where $\varphi$ is a gauge function on $[0, \infty)$. Using this result, strong convergence theorems for common fixed points of a countable family of nonexpansive mappings are established.
\end{abstract}

\section{Introduction}

Let $K$ be a nonempty, closed and convex subset of a real Banach space $E$. Let $T: K \rightarrow K$ be a nonlinear mapping. We denote by $F(T)$ the fixed points set of $T$, that is, $F(T)=\{x \in K: x=T x\}$. A mapping $T$ is called nonexpansive if

$$
\|T x-T y\| \leq\|x-y\|, \quad \forall x, y \in K .
$$

One classical way to study convergence of nonexpansive mappings is to use path convergence for approximating the fixed point of mappings [3, 18, 27]. For any $t \in(0,1)$, we define the mapping $T_{t}: K \rightarrow K$ as follows:

$$
T_{t} x=t u+(1-t) T x, \quad \forall x \in K,
$$

Key Words: Common fixed point, Gauge function, Modified Mann iteration, Nonexpansive mapping, Reflexive Banach space.

2010 Mathematics Subject Classification: 47H09, 47H10

Received: October, 2011.

Accepted: August, 2012. 
where $u \in K$ is fixed. Banach's contraction principle ensures that $T_{t}$ has a unique fixed point $x_{t}$ in $K$ satisfying

$$
x_{t}=t u+(1-t) T x_{t} .
$$

Browder [3] first proved that, if $E$ is a real Hilbert space, then $\left\{x_{t}\right\}$ converges strongly to a fixed point of $T$. Reich [18] showed that Browder's results also valid in a uniformly smooth Banach space. In 2006, Xu [27] proved that Browder's result holds in a reflexive Banach space which has a weakly continuous duality mapping.

On the other hand, Gossez-Lami gave in [9] some geometric properties related to the fixed point theory for nonexpansive mappings. They proved that a space with a weakly continuous duality mapping satisfies Opial's condition [14]. It is also known that all Hilbert spaces and $\ell^{p}(1<p<\infty)$ satisfy the Opial's condition. However, the $L^{p}(1<p<\infty)$ spaces do not unless $p=2$. In this connection, we focus our aim to study a path convergence of (1.2) in a different setting, a real reflexive strictly convex Banach space which has a uniformly Gâteaux differentiable norm concerning a gauge function [4]. We note that our class of Banach spaces includes the spaces $L^{p}, \ell^{p}(1<$ $p<\infty)$ and the Sobolev spaces $W_{m}^{p}(1<p<\infty)$. Moreover, the duality mappings associated with gauge functions also include the generalized and the normalized duality mappings as special cases.

In 1953, Mann [11] introduced the iterative scheme $\left\{x_{n}\right\}$ as follows:

$$
\left\{\begin{array}{l}
x_{0} \in K, \\
x_{n+1}=\alpha_{n} x_{n}+\left(1-\alpha_{n}\right) T x_{n}, \quad \forall n \geq 0,
\end{array}\right.
$$

where $\left\{\alpha_{n}\right\} \subset(0,1)$. If $T$ is a nonexpansive mapping with a fixed point and the control sequence $\left\{\alpha_{n}\right\}$ is chosen such that $\sum_{n=0}^{\infty} \alpha_{n}\left(1-\alpha_{n}\right)=\infty$, then the sequence $\left\{x_{n}\right\}$ defined by (1.3) converges weakly to a fixed point of $T$ (this is also valid in a uniformly convex Banach space with the Fréchet differentiable norm [18]). Since 1953, many authors have constructed and proposed the modified version of algorithm (1.3) in order to get strong convergence results (see $[5,6,10,13,16,24,26,29,30]$ and the references cited therein). Several applications related to the Mann iterative scheme can be found in [17].

Kim-Xu [10] introduced the following modified Mann's iteration as follows:

$$
\left\{\begin{array}{l}
x_{0}=x \in K, \\
y_{n}=\beta_{n} x_{n}+\left(1-\beta_{n}\right) T x_{n}, \\
x_{n+1}=\alpha_{n} u+\left(1-\alpha_{n}\right) y_{n}, \quad \forall n \geq 0,
\end{array}\right.
$$


where $T$ is a nonexpansive mapping of $K$ into itself and $u \in K$ is fixed. They proved, in a uniformly smooth Banach space, that the sequence $\left\{x_{n}\right\}$ defined by (1.4) converges strongly to a fixed point of $T$ if the control sequences $\left\{\alpha_{n}\right\}$ and $\left\{\beta_{n}\right\}$ satisfy appropriate conditions.

Recently, Qin et al. [16] introduced the following iteration:

$$
\left\{\begin{array}{l}
x_{0}=x \in K \\
y_{n}=\beta_{n} x_{n}+\left(1-\beta_{n}\right) W_{n} x_{n}, \\
x_{n+1}=\alpha_{n} u+\left(1-\alpha_{n}\right) y_{n}, \quad \forall n \geq 0,
\end{array}\right.
$$

where $W_{n}$ is the $W$-mapping [20] generated by nonexpansive self mappings $T_{1}, T_{2}, \cdots$ and $\gamma_{1}, \gamma_{2}, \cdots$ and $u \in K$ is fixed. They proved, in a reflexive strictly convex Banach space which has a weakly continuous duality mapping $j_{\varphi}$, that the sequence $\left\{x_{n}\right\}$ defined by (1.5) converges strongly to a common fixed point of $\left\{T_{i}\right\}_{i=1}^{\infty}$ if the control sequences $\left\{\alpha_{n}\right\}$ and $\left\{\beta_{n}\right\}$ satisfy appropriate conditions.

Let $K$ be a nonempty, closed and convex subset of a real Banach space $E$ and $\left\{T_{n}\right\}_{n=1}^{\infty}: K \rightarrow K$ be a sequence of nonexpansive mappings.

Motivated by the works mentioned above, we consider the following modified Mann-type iteration:

$$
\left\{\begin{array}{l}
u, x_{1} \in K \\
y_{n}=\beta_{n} x_{n}+\left(1-\beta_{n}\right) T_{n} x_{n}, \\
x_{n+1}=\alpha_{n} u+\left(1-\alpha_{n}\right) y_{n}, \quad \forall n \geq 1
\end{array}\right.
$$

where $\left\{\alpha_{n}\right\}$ and $\left\{\beta_{n}\right\}$ are real sequences in $(0,1)$.

In this paper, we first prove a path convergence for a nonexpansive mapping in a real reflexive and strictly convex Banach space which has a Gâteaux differentiable norm and admits the duality mapping associated with a gauge function. Then we discuss strong convergence of the modified Mann-type iteration process (1.6) for a countable family of nonexpansive mappings. Our results improve and extend the recent ones announced by many authors.

\section{Preliminaries}

A Banach space $E$ is said to be strictly convex if $\frac{\|x+y\|}{2}<1$ for all $x, y \in E$ with $\|x\|=\|y\|=1$ and $x \neq y$. A Banach space $E$ is called uniformly convex if, for any $\epsilon>0$, there exists $\delta>0$ such that, for any $x, y \in E$ with $\|x\|,\|y\| \leq 1$ and $\|x-y\| \geq \epsilon,\|x+y\| \leq 2(1-\delta)$ holds. The modulus of convexity of $E$ is 
defined by

$$
\delta_{E}(\epsilon)=\inf \left\{1-\left\|\frac{1}{2}(x+y)\right\|:\|x\|,\|y\| \leq 1,\|x-y\| \geq \epsilon\right\}, \quad \forall \epsilon \in[0,2] .
$$

It is known that a Banach space $E$ is uniformly convex if $\delta_{E}(0)=0$ and $\delta_{E}(\epsilon)>0$ for all $0<\epsilon \leq 2$ and every uniformly convex Banach space is strictly convex and reflexive.

Let $S(E)=\{x \in E:\|x\|=1\}$. Then the norm of $E$ is said to be Gâteaux differentiable if

$$
\lim _{t \rightarrow 0} \frac{\|x+t y\|-\|x\|}{t}
$$

exists for any $x, y \in S(E)$. In this case, $E$ is called smooth. The norm of $E$ is said to be uniformly Gateaux differentiable if, for any $y \in S(E)$, the limit is attained uniformly for all $x \in S(E)$.

Let $\rho_{E}:[0, \infty) \rightarrow[0, \infty)$ be the modulus of smoothness of $E$ defined by

$$
\rho_{E}(t)=\sup \left\{\frac{1}{2}(\|x+y\|+\|x-y\|)-1: x \in S(E),\|y\| \leq t\right\} .
$$

A Banach space $E$ is said to be uniformly smooth if $\frac{\rho_{E}(t)}{t} \rightarrow 0$ as $t \rightarrow 0$ (see $[1,7,23]$ for more details).

We recall the following definitions and results which can be found in $[1,4$, 7].

Definition 2.1. A continuous strictly increasing function $\varphi:[0, \infty) \rightarrow[0, \infty)$ is called the gauge function if $\varphi(0)=0$ and $\lim _{t \rightarrow \infty} \varphi(t)=\infty$.

Definition 2.2. Let $E$ be a normed space and $\varphi$ a gauge function. Then the mapping $J_{\varphi}: E \rightarrow 2^{E^{*}}$ defined by

$$
J_{\varphi}(x)=\left\{f^{*} \in E^{*}:\left\langle x, f^{*}\right\rangle=\|x\| \varphi(\|x\|),\left\|f^{*}\right\|=\varphi(\|x\|)\right\}, \quad \forall x \in E,
$$

is called the duality mapping with gauge function $\varphi$.

In particular, if $\varphi(t)=t$, the duality mapping $J_{\varphi}=J$ is called the normalized duality mapping. If $\varphi(t)=t^{q-1}$ for any $q>1$, then the duality mapping $J_{\varphi}=J_{q}$ is called the generalized duality mapping.

It follows from the definition that $J_{\varphi}(x)=\frac{\varphi(\|x\|)}{\|x\|} J(x)$ and $J_{q}(x)=\|x\|^{q-2} J(x)$ for any $q>1$. 
Remark 2.3. [1] For the gauge function $\varphi$, the function $\Phi:[0, \infty) \rightarrow[0, \infty)$ defined by

$$
\Phi(t)=\int_{0}^{t} \varphi(s) d s
$$

is a continuous convex and strictly increasing function on $[0, \infty)$. Therefore, $\Phi$ has a continuous inverse function $\Phi^{-1}$.

Remark 2.4. $[1,7]$ For any $x$ in a Banach space $E, J_{\varphi}(x)=\partial \Phi(\|x\|)$, where $\partial$ denotes the sub-differential.

We know the following subdifferential inequality:

$$
\Phi(\|x+y\|) \leq \Phi(\|x\|)+\left\langle y, j_{\varphi}(x+y)\right\rangle, \quad \forall j_{\varphi}(x+y) \in J_{\varphi}(x+y) .
$$

We also know the following facts (see [1]):

(1) $J_{\varphi}$ is a nonempty, closed and convex set in $E^{*}$ for any $x \in E$.

(2) $J_{\varphi}$ is a function when $E^{*}$ is strictly convex.

(3) If $J_{\varphi}$ is single-valued, then

$$
J_{\varphi}(\lambda x)=\frac{\operatorname{sign}(\lambda) \varphi(\|\lambda x\|)}{\varphi(\|x\|)} J_{\varphi}(x), \quad \forall x \in E, \lambda \in \mathbb{R}
$$

and

$$
\left\langle x-y, J_{\varphi}(x)-J_{\varphi}(y)\right\rangle \geq(\varphi(\|x\|)-\varphi(\|y\|))(\|x\|-\|y\|), \quad \forall x, y \in E .
$$

If $E$ is a smooth Banach space, then $J_{\varphi}$ is single-valued and also denoted by $j_{\varphi}$.

Remark 2.5. [8] Suppose $E$ has a uniformly Gâteaux differentiable norm and admits the duality mapping $j_{\varphi}$. Then $j_{\varphi}$ is uniformly continuous from the norm topology of $E$ to the weak ${ }^{*}$ topology of $E^{*}$ on each bounded subset of $E$.

We next give the definition of Banach limit.

Definition 2.6. Let $\mu$ be a continuous linear functional on $\ell^{\infty}$ and let $\left(a_{0}, a_{1}, \cdots\right) \in$ $\ell^{\infty}$. We write $\mu_{n}\left(a_{n}\right)$ instead of $\mu\left(\left(a_{0}, a_{1}, \cdots\right)\right)$. We call $\mu$ a Banach limit when $\mu$ satisfies $\|\mu\|=\mu_{n}(1)=1$ and $\mu_{n}\left(a_{n}\right)=\mu_{n}\left(a_{n+1}\right)$ for each $\left(a_{0}, a_{1}, \cdots\right) \in \ell^{\infty}$.

For a Banach limit $\mu$, we know that

$$
\liminf _{n \rightarrow \infty} a_{n} \leq \mu_{n}\left(a_{n}\right) \leq \limsup _{n \rightarrow \infty} a_{n}
$$


for all $a=\left(a_{0}, a_{1}, \cdots\right) \in \ell^{\infty}$. Therefore, if $a=\left(a_{0}, a_{1}, \cdots\right) \in \ell^{\infty}, b=$ $\left(b_{0}, b_{1}, \cdots\right) \in \ell^{\infty}$ and $a_{n}-b_{n} \rightarrow 0$ as $n \rightarrow \infty$, then we have $\mu_{n}\left(a_{n}\right)=\mu_{n}\left(b_{n}\right)$ (see $[1,7,23,25])$.

In the sequel, we need the following crucial lemmas:

Lemma 2.7. [21] Let $\left\{x_{n}\right\}$ and $\left\{y_{n}\right\}$ be bounded sequences in a Banach space E such that

$$
x_{n+1}=\left(1-\beta_{n}\right) y_{n}+\beta_{n} x_{n}, \quad \forall n \geq 1,
$$

where $\left\{\beta_{n}\right\}$ is a real sequence in $[0,1]$ with $0<\liminf _{n \rightarrow \infty} \beta_{n} \leq \limsup _{n \rightarrow \infty} \beta_{n}<$ 1. If $\limsup _{n \rightarrow \infty}\left(\left\|y_{n+1}-y_{n}\right\|-\left\|x_{n+1}-x_{n}\right\|\right) \leq 0$, then $\lim _{n \rightarrow \infty}\left\|y_{n}-x_{n}\right\|=0$.

Lemma 2.8. [28] Assume that $\left\{a_{n}\right\}$ is a sequence of nonnegative real numbers such that

$$
a_{n+1} \leq\left(1-\gamma_{n}\right) a_{n}+\gamma_{n} \delta_{n}, \quad \forall n \geq 1,
$$

where $\left\{\gamma_{n}\right\}$ is a sequence in $(0,1)$ and $\left\{\delta_{n}\right\}$ is a sequence in $\mathbb{R}$ such that

(a) $\sum_{n=1}^{\infty} \gamma_{n}=\infty$;

(b) $\limsup _{n \rightarrow \infty} \delta_{n} \leq 0$ or $\sum_{n=1}^{\infty}\left|\gamma_{n} \delta_{n}\right|<\infty$.

Then $\lim _{n \rightarrow \infty} a_{n}=0$.

To deal with a family of mappings, we consider the following condition:

Let $K$ be a subset of a real Banach space $E$ and $\left\{T_{n}\right\}_{n=1}^{\infty}$ be a family of mappings of $K$ such that $\bigcap_{n=1}^{\infty} F\left(T_{n}\right) \neq \emptyset$. Then $\left\{T_{n}\right\}$ is said to satisfy the AKTT-condition [2] if, for any bounded subset $B$ of $K$,

$$
\sum_{n=1}^{\infty} \sup \left\{\left\|T_{n+1} z-T_{n} z\right\|: z \in B\right\}<\infty .
$$

Lemma 2.9. [2] Let $K$ be a nonempty and closed subset of a Banach space $E$ and $\left\{T_{n}\right\}$ be a family of mappings of $K$ into itself which satisfies the AKTTcondition. Then, for any $x \in K,\left\{T_{n} x\right\}$ converges strongly to a point in $K$. Moreover, let the mapping $T$ be defined by

$$
T x=\lim _{n \rightarrow \infty} T_{n} x, \quad \forall x \in K .
$$

Then, for each bounded subset $B$ of $K$,

$$
\lim _{n \rightarrow \infty} \sup \left\{\left\|T z-T_{n} z\right\|: z \in B\right\}=0 .
$$


In the sequel, we write $\left(\left\{T_{n}\right\}, T\right)$ satisfies the AKTT-condition if $\left\{T_{n}\right\}$ satisfies the AKTT-condition and $T$ is defined by Lemma 2.9 with $F(T)=$ $\bigcap_{n=1}^{\infty} F\left(T_{n}\right)$.

Example 2.10. Let $T_{1}, T_{2}, \cdots$, be an infinite family of nonexpansive mappings of $K$ into itself and $\gamma_{1}, \gamma_{2}, \cdots$ be real numbers such that $0<\gamma_{i}<1$ for all $i \in \mathbb{N}$. Moreover, let $W_{n}$ and $W$ be the $W$-mappings [20] generated by $T_{1}, T_{2}, \cdots, T_{n}$ and $\gamma_{1}, \gamma_{2}, \cdots, \gamma_{n}$, and $T_{1}, T_{2}, \cdots$ and $\gamma_{1}, \gamma_{2}, \cdots$. Then $\left(\left\{W_{n}\right\}, W\right)$ satisfies the AKTT-condition (see $\left.[15,20]\right)$.

Example 2.11. Let $T_{1}, T_{2}, \cdots$ be an infinite family of nonexpansive mappings of $K$ into itself. For each $n \in \mathbb{N}$, define the mapping $V_{n}: K \rightarrow K$ by

$$
V_{n} x=\sum_{i=1}^{n} \lambda_{n}^{i} T_{i} x, \quad \forall x \in K,
$$

where $\left\{\lambda_{n}^{i}\right\}$ is a family of nonnegative numbers satisfying the following conditions:

(a) $\sum_{i=1}^{n} \lambda_{n}^{i}=1$ for each $n \in \mathbb{N}$;

(b) $\lambda^{i}:=\lim _{n \rightarrow \infty} \lambda_{n}^{i}>0$ for each $i \in \mathbb{N}$;

(c) $\sum_{n=1}^{\infty} \sum_{i=1}^{n}\left|\lambda_{n+1}^{i}-\lambda_{n}^{i}\right|<\infty$.

Let $V: K \rightarrow K$ be the mapping defined by

$$
V x=\sum_{i=1}^{\infty} \lambda^{i} T_{i} x, \quad \forall x \in K .
$$

Then $\left(\left\{V_{n}\right\}, V\right)$ satisfies the AKTT-condition (see [2]).

\section{Path convergence theorem}

Now, we denote the subset $K^{\prime}$ of $K$ by

$$
K^{\prime}=\left\{x \in K: \mu_{n} \Phi\left(\left\|x_{n}-x\right\|\right)=\inf _{y \in K} \mu_{n} \Phi\left(\left\|x_{n}-y\right\|\right)\right\},
$$

where $\Phi$ is the function defined by (2.1).

Proposition 3.1. [8] Let $K$ be a nonempty, closed and convex subset of a real Banach space $E$ which has a uniformly Gâteaux differentiable norm and admits the duality mapping $j_{\varphi}$. Suppose that $\left\{x_{n}\right\}$ is a bounded sequence of $K$. Let $\mu_{n}$ be a Banach limit and $z \in K$. Then $z \in K^{\prime}$ if and only if

$$
\mu_{n}\left\langle y-z, j_{\varphi}\left(x_{n}-z\right)\right\rangle \leq 0, \quad \forall y \in K
$$


Proposition 3.2. Let $K$ be a nonempty, closed and convex subset of a real reflexive and strictly convex Banach space $E$ which has a uniformly Gâteaux differentiable norm and admits the duality mapping $j_{\varphi}$. Let $T: K \rightarrow K$ be a nonexpansive mapping such that $F(T) \neq \emptyset$. Suppose $\left\{x_{n}\right\}$ is a bounded sequence in $K$ with $\lim _{n \rightarrow \infty}\left\|x_{n}-T x_{n}\right\|=0$. Then $F(T) \cap K^{\prime} \neq \emptyset$.

Proof. Set $g(y)=\mu_{n} \Phi\left(\left\|x_{n}-y\right\|\right)$ for all $y \in K$. Then $g$ is convex and continuous since $\Phi$ is convex and continuous. Further, $g\left(y_{m}\right) \rightarrow \infty$ as $\left\|y_{m}\right\| \rightarrow$ $\infty$ since $\varphi\left(\left\|y_{m}\right\|\right) \rightarrow \infty$ as $\left\|y_{m}\right\| \rightarrow \infty$. Since $E$ is reflexive, by Theorem 1.3.11 in [23], there exists $z \in K$ such that $g(z)=\inf _{y \in K} g(y)$. Hence $K^{\prime}$ is nonempty. Further, $K^{\prime}$ is closed and convex since $g$ is continuous and convex. For any $x \in K^{\prime}$, we have

$$
\begin{aligned}
g(T x) & =\mu_{n} \Phi\left(\left\|x_{n}-T x\right\|\right) \\
& \leq \mu_{n} \Phi\left(\left\|x_{n}-T x_{n}\right\|+\left\|T x_{n}-T x\right\|\right) \\
& \leq \mu_{n} \Phi\left(\left\|x_{n}-x\right\|\right) \\
& =g(x) .
\end{aligned}
$$

Therefore, $T x \in K^{\prime}$ for all $x \in K^{\prime}$.

Let $p \in F(T)$. By Day-James's theorem [12], we know that there exists a unique element $v \in K^{\prime}$ such that

$$
\|p-v\|=\inf _{x \in K^{\prime}}\|p-x\| .
$$

Since $p=T p$ and $T v \in K^{\prime}$, we have

$$
\|p-T v\|=\|T p-T v\| \leq\|p-v\| \leq\|p-T v\| .
$$

It follows that $v=T v$ since $E$ is strictly convex. Hence $v \in F(T) \cap K^{\prime}$. This completes the proof.

Using Propositions 3.1 and 3.2, we next prove a path convergence theorem, which is important to prove our main theorem.

Theorem 3.3. Let $K$ be a nonempty, closed and convex subset of a real reflexive and strictly Banach space $E$ which has a uniformly Gâteaux differentiable norm and admits the duality mapping $j_{\varphi}$. Let $T: K \rightarrow K$ be a nonexpansive such that $F(T) \neq \emptyset$. Fix $u \in K$ and let $t \in(0,1)$. Then the net $\left\{x_{t}\right\}$ defined by (1.2) converges strongly as $t \rightarrow 0$ to a fixed point $p$ of $T$ which solves the variational inequality:

$$
\left\langle u-p, j_{\varphi}(w-p)\right\rangle \leq 0, \quad \forall w \in F(T) .
$$


Proof. First, we prove that the solution of variational inequality (3.1) is unique. Suppose that $p, q \in F(T)$ satisfy (3.1). Then we have

$$
\left\langle u-p, j_{\varphi}(q-p)\right\rangle \leq 0, \quad\left\langle u-q, j_{\varphi}(p-q)\right\rangle \leq 0 .
$$

Adding the above inequalities, we obtain

$$
\left\langle p-q, j_{\varphi}(p-q)\right\rangle \leq 0
$$

which implies that

$$
\|p-q\| \varphi(\|p-q\|) \leq 0
$$

and so $p=q$.

Next, we prove that $\left\{x_{t}\right\}$ is bounded in $K$. For any $w \in F(T)$, we see that

$$
\begin{aligned}
& \left\|x_{t}-w\right\| \varphi\left(\left\|x_{t}-w\right\|\right) \\
= & \left\langle x_{t}-w, j_{\varphi}\left(x_{t}-w\right)\right\rangle \\
= & t\left\langle u-w, j_{\varphi}\left(x_{t}-w\right)\right\rangle+(1-t)\left\langle T x_{t}-w, j_{\varphi}\left(x_{t}-w\right)\right\rangle \\
\leq & t\left\langle u-w, j_{\varphi}\left(x_{t}-w\right)\right\rangle+(1-t)\left\|x_{t}-w\right\| \varphi\left(\left\|x_{t}-w\right\|\right),
\end{aligned}
$$

which implies

$$
\begin{aligned}
\left\|x_{t}-w\right\| \varphi\left(\left\|x_{t}-w\right\|\right) & \leq\left\langle u-w, j_{\varphi}\left(x_{t}-w\right)\right\rangle \\
& \leq\|u-w\| \varphi\left(\left\|x_{t}-w\right\|\right) .
\end{aligned}
$$

Hence $\left\|x_{t}-w\right\| \leq\|u-w\|$ and, consequently, $\left\{x_{t}\right\}$ is bounded. So is $\left\{T x_{t}\right\}$. We see that

$$
\left\|x_{t}-T x_{t}\right\|=t\left\|u-T x_{t}\right\| \rightarrow 0 \quad(t \rightarrow 0) .
$$

Since $E$ is reflexive, $\left\{x_{t}\right\}$ has a weakly convergent subsequence $\left\{x_{t_{n}}\right\}$. Thus $\left\{x_{t_{n}}\right\}$ is bounded. Putting $x_{n}:=x_{t_{n}}$, in particular, we also have

$$
\left\|x_{n}-T x_{n}\right\| \rightarrow 0 \quad(n \rightarrow \infty) .
$$

By Proposition 3.2, since $\left\{x_{n}\right\}$ is bounded, there exists $p \in F(T)$ such that

$$
\mu_{n} \Phi\left(\left\|x_{n}-p\right\|\right)=\inf _{y \in K} \mu_{n} \Phi\left(\left\|x_{n}-y\right\|\right) .
$$

It follows from Proposition 3.1 that

$$
\mu_{n}\left\langle y-p, j_{\varphi}\left(x_{n}-p\right)\right\rangle \leq 0, \quad \forall y \in K .
$$

Since $u \in K$, in particular, we have

$$
\mu_{n}\left\langle u-p, j_{\varphi}\left(x_{n}-p\right)\right\rangle \leq 0 .
$$


Observe that

$$
\Phi(\|y\|)=\int_{0}^{\|y\|} \varphi(s) d s \leq\|y\| \varphi(\|y\|) .
$$

It follows from (3.2) and (3.3) that

$$
\mu_{n} \Phi\left(\left\|x_{n}-p\right\|\right) \leq \mu_{n}\left\langle u-p, j_{\varphi}\left(x_{n}-p\right)\right\rangle \leq 0
$$

and hence

$$
\mu_{n} \Phi\left(\left\|x_{n}-p\right\|\right)=0 .
$$

Since $\Phi$ is continuous, there exists a subsequence $\left\{x_{n_{k}}\right\}$ of $\left\{x_{n}\right\}$ such that $\left\{x_{n_{k}}\right\}$ converges strongly to $p$. Let $\left\{x_{n_{j}}\right\}$ be another subsequence of $\left\{x_{n}\right\}$ such that $x_{n_{j}} \rightarrow q$ as $j \rightarrow \infty$. From (3.4), we have

$$
\mu_{j} \Phi\left(\left\|x_{n_{j}}-p\right\|\right)=\Phi(\|q-p\|)=0
$$

and so $p=q$. Therefore, the sequence $\left\{x_{n}\right\}$ converges strongly to a fixed point $p$ of $T$.

Next, we prove that $p \in F(T)$ is a solution to the variational inequality (3.1). For any $w \in F(T)$, we see that

$$
\begin{aligned}
\left\|x_{n}-w\right\| \varphi\left(\left\|x_{n}-w\right\|\right)= & \left\langle x_{n}-w, j_{\varphi}\left(x_{n}-w\right)\right\rangle \\
= & t_{n}\left\langle u-p, j_{\varphi}\left(x_{n}-w\right)\right\rangle+t_{n}\left\langle p-x_{n}, j_{\varphi}\left(x_{n}-w\right)\right\rangle \\
& +t_{n}\left\langle x_{n}-w, j_{\varphi}\left(x_{n}-w\right)\right\rangle \\
& +\left(1-t_{n}\right)\left\langle T x_{n}-w, j_{\varphi}\left(x_{n}-w\right)\right\rangle \\
\leq & t_{n}\left\langle u-p, j_{\varphi}\left(x_{n}-w\right)\right\rangle+t_{n}\left\|x_{n}-p\right\| \varphi\left(\left\|x_{n}-w\right\|\right) \\
& +t_{n}\left\|x_{n}-w\right\| \varphi\left(\left\|x_{n}-w\right\|\right) \\
& +\left(1-t_{n}\right)\left\|x_{n}-w\right\| \varphi\left(\left\|x_{n}-w\right\|\right) \\
= & t_{n}\left\langle u-p, j_{\varphi}\left(x_{n}-w\right)\right\rangle+t_{n}\left\|x_{n}-p\right\| \varphi\left(\left\|x_{n}-w\right\|\right) \\
& +\left\|x_{n}-w\right\| \varphi\left(\left\|x_{n}-w\right\|\right) .
\end{aligned}
$$

This implies that

$$
\left\langle u-p, j_{\varphi}\left(w-x_{n}\right)\right\rangle \leq\left\|x_{n}-p\right\| \varphi\left(\left\|x_{n}-w\right\|\right) .
$$

Since $j_{\varphi}$ is norm-weak* uniformly continuous on bounded subsets of $E$, we have

$$
\left\langle u-p, j_{\varphi}\left(w-x_{n}\right)\right\rangle \rightarrow\left\langle u-p, j_{\varphi}(w-p)\right\rangle \quad(n \rightarrow \infty) .
$$

Thus, taking the limit as $n \rightarrow \infty$ in both sides of (3.5), we get

$$
\left\langle u-p, j_{\varphi}(w-p)\right\rangle \leq 0, \quad \forall w \in F(T) .
$$


Finally, we prove that $x_{t} \rightarrow p$ as $t \rightarrow 0$. To this end, let $\left\{x_{s_{n}}\right\}$ be another subsequence of $\left\{x_{t}\right\}$ such that $x_{s_{n}} \rightarrow p^{\prime}$ as $n \rightarrow \infty$. We have to show that $p=p^{\prime}$. For any $w \in F(T)$, we have

$$
\begin{aligned}
\left\langle T x_{t}-x_{t}, j_{\varphi}\left(x_{t}-w\right)\right\rangle & =\left\langle T x_{t}-w, j_{\varphi}\left(x_{t}-w\right)\right\rangle+\left\langle w-x_{t}, j_{\varphi}\left(x_{t}-w\right)\right\rangle \\
& \leq\left\|x_{t}-w\right\| \varphi\left(\left\|x_{t}-w\right\|\right)+\left\langle w-x_{t}, j_{\varphi}\left(x_{t}-w\right)\right\rangle \\
& =\left\langle x_{t}-w, j_{\varphi}\left(x_{t}-w\right)\right\rangle+\left\langle w-x_{t}, j_{\varphi}\left(x_{t}-w\right)\right\rangle \\
& =0 .
\end{aligned}
$$

On the other hand, since

$$
x_{t}-T x_{t}=\frac{t}{1-t}\left(u-x_{t}\right),
$$

we have

$$
\left\langle x_{t}-u, j_{\varphi}\left(x_{t}-w\right)\right\rangle \leq 0, \quad \forall w \in F(T) .
$$

In particular, we have

$$
\left\langle x_{t_{n}}-u, j_{\varphi}\left(x_{t_{n}}-p^{\prime}\right)\right\rangle \leq 0
$$

and

$$
\left\langle x_{s_{n}}-u, j_{\varphi}\left(x_{s_{n}}-p\right)\right\rangle \leq 0
$$

or, equivalently,

$$
\left\|x_{t_{n}}-p^{\prime}\right\| \varphi\left(\left\|x_{t_{n}}-p^{\prime}\right\|\right)+\left\langle p^{\prime}-u, j_{\varphi}\left(x_{t_{n}}-p^{\prime}\right)\right\rangle \leq 0
$$

and

$$
\left\|x_{s_{n}}-p\right\| \varphi\left(\left\|x_{s_{n}}-p\right\|\right)+\left\langle p-u, j_{\varphi}\left(x_{s_{n}}-p\right)\right\rangle \leq 0 .
$$

Taking the limit as $n \rightarrow \infty$, since $\varphi$ is continuous and $j_{\varphi}$ is norm-to-weak ${ }^{*}$ uniformly continuous on bounded subsets of $E$, we obtain

$$
\left\|p-p^{\prime}\right\| \varphi\left(\left\|p-p^{\prime}\right\|\right)+\left\langle p^{\prime}-u, j_{\varphi}\left(p-p^{\prime}\right)\right\rangle \leq 0
$$

and

$$
\left\|p^{\prime}-p\right\| \varphi\left(\left\|p^{\prime}-p\right\|\right)+\left\langle p-u, j_{\varphi}\left(p^{\prime}-p\right)\right\rangle \leq 0 .
$$

Summing the above inequalities, we also have

$$
2\left\|p-p^{\prime}\right\| \varphi\left(\left\|p-p^{\prime}\right\|\right)+\left\langle p^{\prime}-p, j_{\varphi}\left(p-p^{\prime}\right)\right\rangle \leq 0 .
$$

This implies that

$$
\left\langle p-p^{\prime}, j_{\varphi}\left(p-p^{\prime}\right)\right\rangle \leq 0
$$

and hence $p=p^{\prime}$. Therefore, $\left\{x_{t}\right\}$ converges strongly to a fixed point of $T$. This completes the proof. 


\section{Strong convergence theorems}

In this section, using Theorem 3.3, we prove a strong convergence theorem in a real reflexive and strictly convex Banach space which has a uniformly Gâteaux differentiable norm and admits the duality mapping $j_{\varphi}$, where $\varphi$ is a gauge function on $[0, \infty)$.

Theorem 4.1. Let $K$ be a nonempty closed and convex subset of a real reflexive and strictly convex Banach space $E$ which has a uniformly Gâteaux differentiable norm and admits the duality mapping $j_{\varphi}$. Let $\left\{T_{n}\right\}_{n=1}^{\infty}: K \rightarrow K$ be a sequence of nonexpansive mappings such that $F:=\bigcap_{n=1}^{\infty} F\left(T_{n}\right) \neq \emptyset$. Let $u \in K$ be fixed. Let $\left\{\alpha_{n}\right\}$ and $\left\{\beta_{n}\right\}$ be real sequences in $(0,1)$ such that
(a) $\lim _{n \rightarrow \infty} \alpha_{n}=0$;
(b) $\sum_{n=1}^{\infty} \alpha_{n}=\infty$;
(c) $0<\liminf _{n \rightarrow \infty} \beta_{n} \leq \lim \sup _{n \rightarrow \infty} \beta_{n}<1$.

If $\left(\left\{T_{n}\right\}, T\right)$ satisfies the AKTT-condition, then the sequences $\left\{x_{n}\right\}$ and $\left\{y_{n}\right\}$ defined by (1.6) converge strongly to $p \in F$ which also solves the variational inequality (3.1).

Proof. First, we see that the sequences $\left\{x_{n}\right\}$ and $\left\{y_{n}\right\}$ is bounded. In fact, for any $w \in F$, we have

$$
\left\|y_{n}-w\right\| \leq \beta_{n}\left\|x_{n}-w\right\|+\left(1-\beta_{n}\right)\left\|T_{n} x_{n}-w\right\| \leq\left\|x_{n}-w\right\|
$$

and so

$$
\begin{aligned}
\left\|x_{n+1}-w\right\| \leq & \alpha_{n}\|u-w\|+\left(1-\alpha_{n}\right)\left\|y_{n}-w\right\| \\
\leq & \alpha_{n}\|u-w\|+\left(1-\alpha_{n}\right)\left\|x_{n}-w\right\| \\
& \leq \max \left\{\left\|x_{n}-w\right\|,\|u-w\|\right\} .
\end{aligned}
$$

Hence the sequence $\left\{x_{n}\right\}$ is bounded by induction and so is $\left\{y_{n}\right\}$.

Next, we show that

$$
\lim _{n \rightarrow \infty}\left\|x_{n+1}-x_{n}\right\|=0
$$

Putting $l_{n}=\frac{x_{n+1}-\beta_{n} x_{n}}{1-\beta_{n}}$, we get

$$
x_{n+1}=\left(1-\beta_{n}\right) l_{n}+\beta_{n} x_{n}, \quad \forall n \geq 1 .
$$


Thus we have

$$
\begin{aligned}
& l_{n+1}-l_{n} \\
= & \frac{\alpha_{n+1} u+\left(1-\alpha_{n+1}\right) y_{n+1}-\beta_{n+1} x_{n+1}}{1-\beta_{n+1}}-\frac{\alpha_{n} u+\left(1-\alpha_{n}\right) y_{n}-\beta_{n} x_{n}}{1-\beta_{n}} \\
= & \frac{\alpha_{n+1}\left(u-y_{n+1}\right)}{1-\beta_{n+1}}-\frac{\alpha_{n}\left(u-y_{n}\right)}{1-\beta_{n}}+T_{n+1} x_{n+1}-T_{n} x_{n},
\end{aligned}
$$

which implies

$$
\begin{aligned}
& \left\|l_{n+1}-l_{n}\right\| \\
\leq & \frac{\alpha_{n+1}}{1-\beta_{n+1}}\left\|u-y_{n+1}\right\|+\frac{\alpha_{n}}{1-\beta_{n}}\left\|u-y_{n}\right\|+\left\|x_{n+1}-x_{n}\right\|+\left\|T_{n+1} x_{n}-T_{n} x_{n}\right\| \\
\leq & \frac{\alpha_{n+1}}{1-\beta_{n+1}}\left\|u-y_{n+1}\right\|+\frac{\alpha_{n}}{1-\beta_{n}}\left\|u-y_{n}\right\|+\left\|x_{n+1}-x_{n}\right\|+\sup _{z \in\left\{x_{n}\right\}}\left\|T_{n+1} z-T_{n} z\right\| .
\end{aligned}
$$

Since $\left\{T_{n}\right\}$ satisfies the AKTT-condition, it follows from the conditions (a) and (c) that

$$
\limsup _{n \rightarrow \infty}\left(\left\|l_{n+1}-l_{n}\right\|-\left\|x_{n+1}-x_{n}\right\|\right) \leq 0
$$

By Lemma 2.7, we also obtain

$$
\lim _{n \rightarrow \infty}\left\|l_{n}-x_{n}\right\|=0 .
$$

Since

$$
x_{n+1}-x_{n}=\left(1-\beta_{n}\right)\left(l_{n}-x_{n}\right),
$$

we have

$$
\left\|x_{n+1}-x_{n}\right\|=\left(1-\beta_{n}\right)\left\|l_{n}-x_{n}\right\| \rightarrow 0 \quad(n \rightarrow \infty) .
$$

On the other hand, we see that

$$
\left\|x_{n+1}-y_{n}\right\|=\alpha_{n}\left\|u-y_{n}\right\| \rightarrow 0 \quad(n \rightarrow \infty) .
$$

Combining (4.1) and (4.2) we obtain

$$
\lim _{n \rightarrow \infty}\left\|x_{n}-y_{n}\right\|=0
$$

Noting that

$$
\begin{aligned}
\left\|x_{n}-T_{n} x_{n}\right\| & \leq\left\|x_{n}-y_{n}\right\|+\left\|y_{n}-T_{n} x_{n}\right\| \\
& =\left\|x_{n}-y_{n}\right\|+\beta_{n}\left\|x_{n}-T_{n} x_{n}\right\|,
\end{aligned}
$$


from (4.3) and the condition (c), we have

$$
\lim _{n \rightarrow \infty}\left\|x_{n}-T_{n} x_{n}\right\|=0 .
$$

Further, we have

$$
\begin{aligned}
\left\|x_{n}-T x_{n}\right\| & \leq\left\|x_{n}-T_{n} x_{n}\right\|+\left\|T_{n} x_{n}-T x_{n}\right\| \\
& \leq\left\|x_{n}-T_{n} x_{n}\right\|+\sup _{z \in\left\{x_{n}\right\}}\left\|T_{n} z-T z\right\| .
\end{aligned}
$$

Thus, by Lemma 2.9 and (4.4), we have

$$
\lim _{n \rightarrow \infty}\left\|x_{n}-T x_{n}\right\|=0 .
$$

Since $T$ is nonexpansive, by Theorem 3.3, we know that the net $\left\{x_{t}\right\}$ generated by (1.2) converges strongly to a fixed point $p \in F(T)=F$ which also solves the variational inequality (3.1).

Next, we prove that

$$
\limsup _{n \rightarrow \infty}\left\langle u-p, j_{\varphi}\left(x_{n}-p\right)\right\rangle \leq 0 .
$$

Observe that

$$
\begin{aligned}
& \left\|x_{t}-x_{n}\right\| \varphi\left(\left\|x_{t}-x_{n}\right\|\right) \\
= & t\left\langle u-x_{n}, j_{\varphi}\left(x_{t}-x_{n}\right)\right\rangle+(1-t)\left\langle T x_{t}-x_{n}, j_{\varphi}\left(x_{t}-x_{n}\right)\right\rangle \\
= & t\left\langle p-x_{t}, j_{\varphi}\left(x_{t}-x_{n}\right)\right\rangle+t\left\langle u-p, j_{\varphi}\left(x_{t}-x_{n}\right)\right\rangle \\
& +t\left\langle x_{t}-x_{n}, j_{\varphi}\left(x_{t}-x_{n}\right)\right\rangle+(1-t)\left\langle T x_{t}-T x_{n}, j_{\varphi}\left(x_{t}-x_{n}\right)\right\rangle \\
& +(1-t)\left\langle T x_{n}-x_{n}, j_{\varphi}\left(x_{t}-x_{n}\right)\right\rangle \\
\leq & t\left\|p-x_{t}\right\| \varphi\left(\left\|x_{t}-x_{n}\right\|\right)+t\left\langle u-p, j_{\varphi}\left(x_{t}-x_{n}\right)\right\rangle \\
& +\left\|x_{t}-x_{n}\right\| \varphi\left(\left\|x_{t}-x_{n}\right\|\right)+\left\|T x_{n}-x_{n}\right\| \varphi\left(\left\|x_{t}-x_{n}\right\|\right) .
\end{aligned}
$$

Therefore, it follows that

$$
\left\langle u-p, j_{\varphi}\left(x_{n}-x_{t}\right)\right\rangle \leq \frac{\left\|T x_{n}-x_{n}\right\| \varphi\left(\left\|x_{t}-x_{n}\right\|\right)}{t}+\left\|x_{t}-p\right\| \varphi\left(\left\|x_{t}-x_{n}\right\|\right) .
$$

Using (4.5) and taking the limit as $n \rightarrow \infty$ first and then, as $t \rightarrow 0$, the inequality (4.6) becomes

$$
\limsup _{t \rightarrow 0} \limsup _{n \rightarrow \infty}\left\langle u-p, j_{\varphi}\left(x_{n}-x_{t}\right)\right\rangle \leq 0 .
$$


Since $j_{\varphi}$ is norm-weak* uniformly continuous on bounded sets,

$$
\left\langle u-p, j_{\varphi}\left(x_{n}-x_{t}\right)\right\rangle \rightarrow\left\langle u-p, j_{\varphi}\left(x_{n}-p\right)\right\rangle \quad(t \rightarrow 0) .
$$

We see that

$\left\langle u-p, j_{\varphi}\left(x_{n}-p\right)\right\rangle=\left\langle u-p, j_{\varphi}\left(x_{n}-x_{t}\right)\right\rangle+\left\langle u-p, j_{\varphi}\left(x_{n}-p\right)-j_{\varphi}\left(x_{n}-x_{t}\right)\right\rangle$.

By the uniform continuity of $j_{\varphi}$, we can interchange the two limits above and deduce that

$$
\limsup _{n \rightarrow \infty}\left\langle u-p, j_{\varphi}\left(x_{n}-p\right)\right\rangle \leq 0 .
$$

Finally, we prove that $x_{n} \rightarrow p$ as $n \rightarrow \infty$. Observe that

$$
\begin{aligned}
\Phi\left(\left\|y_{n}-p\right\|\right) & =\Phi\left(\left\|\beta_{n}\left(x_{n}-p\right)+\left(1-\beta_{n}\right)\left(T_{n} x_{n}-p\right)\right\|\right) \\
& \leq \beta_{n} \Phi\left(\left\|x_{n}-p\right\|\right)+\left(1-\beta_{n}\right) \Phi\left(\left\|T_{n} x_{n}-p\right\|\right) \\
& \leq \Phi\left(\left\|x_{n}-p\right\|\right) .
\end{aligned}
$$

Form (2.2), it follows that

$$
\begin{aligned}
\Phi\left(\left\|x_{n+1}-p\right\|\right) & =\Phi\left(\left\|\alpha_{n}(u-p)+\left(1-\alpha_{n}\right)\left(y_{n}-p\right)\right\|\right) \\
& \leq \Phi\left(\left(1-\alpha_{n}\right)\left\|y_{n}-p\right\|\right)+\alpha_{n}\left\langle u-p, j_{\varphi}\left(x_{n+1}-p\right)\right\rangle \\
& \leq\left(1-\alpha_{n}\right) \Phi\left(\left\|x_{n}-p\right\|\right)+\alpha_{n}\left\langle u-p, j_{\varphi}\left(x_{n+1}-p\right)\right\rangle .
\end{aligned}
$$

Applying Lemma 2.8, we have $\Phi\left(\left\|x_{n}-p\right\|\right) \rightarrow 0$ as $n \rightarrow \infty$ by the condition (b) and (4.8). Hence $x_{n} \rightarrow p$ as $n \rightarrow \infty$ since $\Phi$ is continuous. Moreover, the sequence $\left\{y_{n}\right\}$ also strongly converges to $p$. This completes the proof.

Remark 4.2. From Examples 2.10 and 2.11, the ordered pair $\left(\left\{T_{n}\right\}, T\right)$ in Theorem 4.1 can be replaced by $\left(\left\{W_{n}\right\}, W\right)$ and $\left(\left\{V_{n}\right\}, V\right)$.

Remark 4.3. Theorem 4.1 mainly improves and extends the results of Kim$\mathrm{Xu}[10]$ in the following aspects: [10];

(1) we relax the restrictions imposed on the parameters in Theorem 1 of

(2) we extend Theorem 1 of [10] from a single nonexpansive mapping to an infinite family of nonexpansive mappings;

(3) we extend Theorem 1 of [10] from a uniformly smooth Banach space to a much more general setting.

Remark 4.4. If $f: K \rightarrow K$ is a contraction and we replace $u$ by $f\left(x_{n}\right)$ in the recursion formula (1.6), we can obtain the so-called viscosity iteration method (see $[22])$. 
Remark 4.5. Theorem 3.3 and Theorem 4.1 can be applied to the spaces $L^{p}$, $\ell^{p}(1<p<\infty)$, the Sobolev spaces $W_{m}^{p}(1<p<\infty)$ and Hilbert spaces. Moreover, our results hold for a Banach space which has the generalized duality mapping $j_{q}(q>1)$ and the normalized the duality mapping $j$.

Acknowledgement. The first author was supported by the Thailand Research Fund, the Commission on Higher Education, and University of Phayao under Grant MRG5580016.

\section{References}

[1] R.P. Agarwal, D. O'Regan, D.R. Sahu, Fixed Point Theory for Lipschitziantype Mappings with Applications, Springer, New York (2009).

[2] K. Aoyama, Y. Kimura, W. Takahashi, M. Toyoda, Approximation of common fixed points of a countable family of nonexpansive mappings in a Banach space, Nonlinear Anal. 67 (2007) 2350-2360.

[3] F.E. Browder, Fixed point theorems for noncompact mappings in Hilbert spaces, Proc. Natl. Acad. Sci. USA 53 (1965) 1272-1276.

[4] F.E. Browder, Convergence theorems for sequences of nonlinear operators in Banach spaces, Math. Z. 100 (1967) 201-225.

[5] S.S. Chang, Viscosity approximation methods for a finite family of nonexpansive mappings in Banach spaces, J. Math. Anal. Appl. 323 (2006) 1402-1416.

[6] Y.J. Cho, S.M. Kang, X. Qin, Approximation of common fixed points of an infinite family of nonexpansive mappings in Banach spaces, Comput. Math. Appl. 56 (2008) 2058-2064.

[7] C.E. Chidume, Geometric Properties of Banach Spaces and Nonlinear Iterations, in: Springer Lecture Notes Series, 2009.

[8] P. Cholamjiak, S. Suantai, Viscosity approximation methods for a nonexpansive semigroup in Banach spaces with gauge functions, J. Glob. Optim. (2011), doi: 10.1007/s10898-011-9756-4.

[9] J.P. Gossez, D.E. Lami, Some geometric properties related to the fixed point theory for nonexpansive mappings, Pacific J. Math. 40 (1972) 565-573.

[10] T.H. Kim, H.K. Xu, Strong convergence of modified Mann iterations, Nonlinear Anal. 61 (2005) 51-60. 
[11] W.R. Mann, Mean value methods in iteration, Proc. Am. Math. Soc. 4 (1953) 506-510.

[12] R.E. Megginson, An Introduction to Banach Space Theory, Springer, New York (1998).

[13] A. Moudafi, Viscosity approximation methods for fixed point problems, J. Math. Anal. Appl. 241 (2000) 46-55.

[14] Z. Opial, Weak convergence of successive approximations for nonexpansive mappings, Bull. Amer. Math. Soc. 73 (1967) 591-597.

[15] J.W. Peng, J.C. Yao, A viscosity approximation scheme for system of equilibrium problems, nonexpansive mappings and monotone mappings, Nonlinear Anal. 71 (2009) 6001-6010.

[16] X. Qin, Y.J. Cho, J.I. Kang, S.M. Kang, Strong convergence theorems for an infinite family of nonexpansive mappings in Banach spaces, J. Comput. Appl. Math. 230 (2009) 121-127.

[17] T.-L. Radulescu, V. Radulescu, T. Andreescu, Problems in Real Analysis: Advanced Calculus on the Real Axis, Springer, New York, 2009.

[18] S. Reich, Strong convergence theorems for resolvents of accretive operators in Banach spaces, J. Math. Anal. Appl. 75 (1980) 287-292.

[19] S. Reich, Weak convergence theorems for nonexpansive mappings in Banach spaces, J. Math. Anal. Appl. 67 (1979) 274-276.

[20] K. Shimoji, W. Takahashi, Strong convergence to common fixed points of infinite nonexpansive mappings and applications, Taiwan. J. Math. 5 (2001) 387404.

[21] T. Suzuki, Strong convergence of Krasnoselskii and Manns type sequences for one parameter nonexpansive semigroups without Bochner integrals, J. Math. Anal. Appl. 305 (2005) 227-239.

[22] T. Suzuki, Moudafi's viscosity approximations with Meir-Keeler contractions, J. Math. Anal. Appl. 325 (2007) 342-352.

[23] W. Takahashi, Nonlinear Function Analysis, Yokahama Publishers, Yokahama (2000).

[24] W. Takahashi, Y. Takeuchi, R. Kubota, Strong convergence theorems by hybrid methods for families of nonexpansive mappings in Hilbert spaces, J. Math. Anal. Appl. 341 (2008) 276-286. 
[25] W. Takahashi, Y. Ueda, On Reich's strong convergence for resolvents of accretive operators, J. Math. Anal. Appl. 104 (1984) 546-553.

[26] R. Wangkeeree, N. Petrot, R. Wangkeeree, The general iterative methods for nonexpansive mappings in Banach spaces, J. Glob. Optim., doi 10.1007/s10898010-9617-6.

[27] H.K. Xu, Strong convergence of an iterative method for nonexpansive and accretive operators, J. Math. Anal. Appl. 314 (2006) 631-643.

[28] H.K. Xu, Iterative algorithms for nonlinear operators, J. Lond. Math. Soc. 66 (2002) 240-256.

[29] Y. Yao, R. Chen, J.C. Yao, Strong convergence and certain control conditions for modified Mann iteration, Nonlinear Anal. 68 (2008) 1687-1693.

[30] Y. Yao, J.C. Yao, H. Zhou, Approximation methods for common fixed points of infinite countable family of nonexpansive mappings, Comput. Math. Appl. 53 (2007) 1380-1389.

Prasit Cholamjiak,

School of Science,

University of Phayao,

Phayao 56000, Thailand.

Email: prasitch2008@yahoo.com

Yeol Je Cho,

Department of Mathematics Education and the RINS,

Gyeongsang National University,

Jinju 660-701, Republic of Korea.

Email: yjcho@gnu.ac.kr

Suthep Suantai,

Department of Mathematics,

Faculty of Science,

Chiang Mai University,

Chiang Mai 50200, Thailand.

Email: scmti005@chiangmai.ac.th 